\title{
Arthroscopic Removal of a Screwdriver Fragment in a Dog 6 Years after Femoral Fracture Repair
}

\author{
Massimo Petazzoni ${ }^{1}$ \\ ${ }^{1}$ Department of Orthopaedic, Clinica Veterinaria Milano Sud, \\ Peschiera Borromeo, Milan, Italy \\ VCOT Open 2020;3:e23-e27.
}

\begin{abstract}
Address for correspondence Massimo Petazzoni, DVM, Department of Orthopaedic, Clinica Veterinaria Milano Sud, Via della Liberazione 26, Peschiera Borromeo, MI 20068, Italy

(e-mail: massimo.petazzoni@cvmilanosud.it).
\end{abstract}

\begin{abstract}
Keywords

- Complication

- foreign body

- tibiofemoral joint

- canine

Objective The aim of this study was to report an unusual case of an iatrogenic foreign body within the stifle joint, removed 6 years after distal femoral fracture repair in a small dog. Acute lameness was caused by a migrating screwdriver fragment, which had been lost during initial fracture repair and which did not cause clinical signs for years until dislodged inside the joint.

Case Report A male Jack Russell, 7 years of age, underwent plate and screws fixation to treat a Salter-Harris type I fracture at the age of 9 months. Seven months after surgery, partial implant removal was performed. Six years after fracture repair, the dog presented for a non-weight-bearing lameness and pain on the previously operated stifle joint, which occurred suddenly while walking on the leash. Radiographs revealed a small intra-articular metallic radiodense foreign body. Arthroscopy was used to remove the migrating broken-tip of a screwdriver from the medial aspect of the tibiofemoral joint. Retrieving the foreign body led to the immediate resolution of clinical signs without complications.

Conclusion By reporting this case of a forgotten foreign body retained inside a stifle joint for 6 years, we aim to illustrate the potential risk of leaving a piece of metal inside the body. A high index of suspicion for such complications should be kept a long time after surgery. Arthroscopy was a useful first-line tool for efficiently and mini-invasively treating this unusual problem.
\end{abstract}

\section{Introduction}

There are reports of joint foreign bodies caused by penetration of the skin or after arthroscopy in both human or veterinary medicine. ${ }^{1-10}$ Stifle joints arthroscopic removal of bullets, metal fragments, needles, glass and plastic objects have been reported. . $^{3,10-12}$ When being lodged intra-articular, foreign bodies can cause lesions of the cartilage, menisci or ligaments. ${ }^{10-14}$

We report an unusual case in which arthroscopy was used to remove a broken and previously lost tip of a screwdriver from the medial aspect of the tibiofemoral joint of a small dog. There seems no similar report in dogs of a metallic foreign body penetrating and lodging within the joint without, evidently, causing any clinical signs for several years.

\section{Case Report}

A 7-year-old male Jack Russell Terrier was presented for a non-weight-bearing right hind-limb lameness of 1-week duration, right stifle pain and joint swelling. Sudden onset of lameness occurred while the dog was walking on a leash without a history of trauma or excessive exercise.

The dog's medical history included a right distal femoral Salter-Harris type I fracture, which occurred when the dog was 9 months old ( - Fig. 1A, B). Fracture repair was performed using one locking plate and six screws (PAX $2.4 \mathrm{~mm}$ received

October 20, 2019

accepted

February 24, 2020
DOI https://doi.org/

10.1055/s-0040-1709451. ISSN 2625-2325. (c) 2020 Georg Thieme Verlag KG
Stuttgart · New York

License terms

(c) (1) 


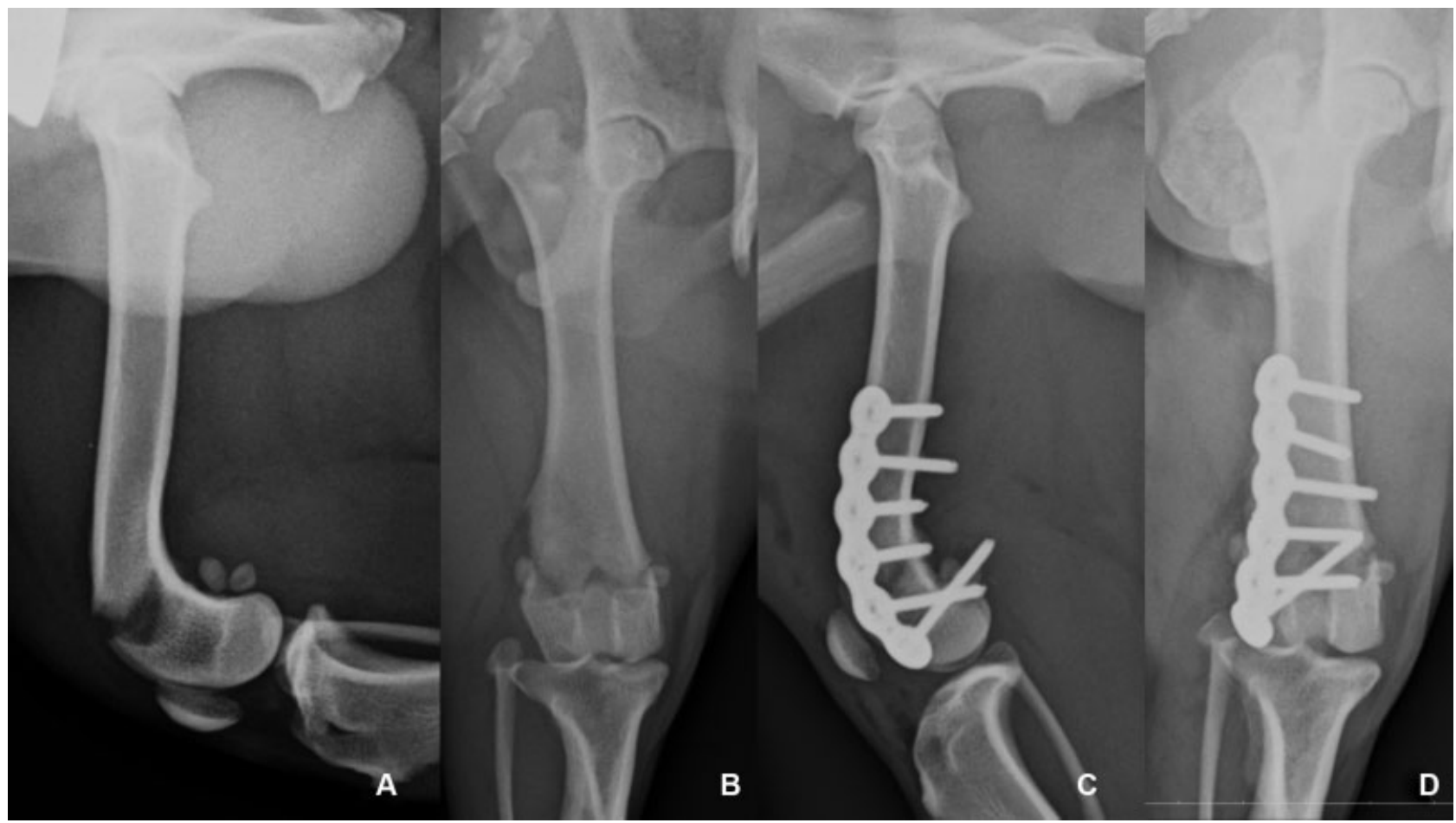

Fig. 1 Jack Russell Terrier, male, 9 months old. Mediolateral view and craniocaudal view of the right femur (A, B). Salter-Harris type I fracture was diagnosed. Fracture repair was performed using a 6-hole locking plate (C, D).

Polyaxial Locking Reconstruction Plate System, Securos Surgical, Massachusetts, United States) (-Fig. 1C, D). At 3 months, fracture healing was confirmed radiographically (-Fig. 2A, B). At 7 months, a radiographic examination showed some reabsorption of bone, secondary to alleged stress shielding (-Fig. 2C, D). A radio-dense fragment of $\sim 2.5 \times 2 \mathrm{~mm}$ dimension was noticed at the level of the distal pole of the patella in the mediolateral view and at the level of the lateral femoral condyle on the craniocaudal view (-Fig. 2C, D, arrows). Plate and screws were removed.

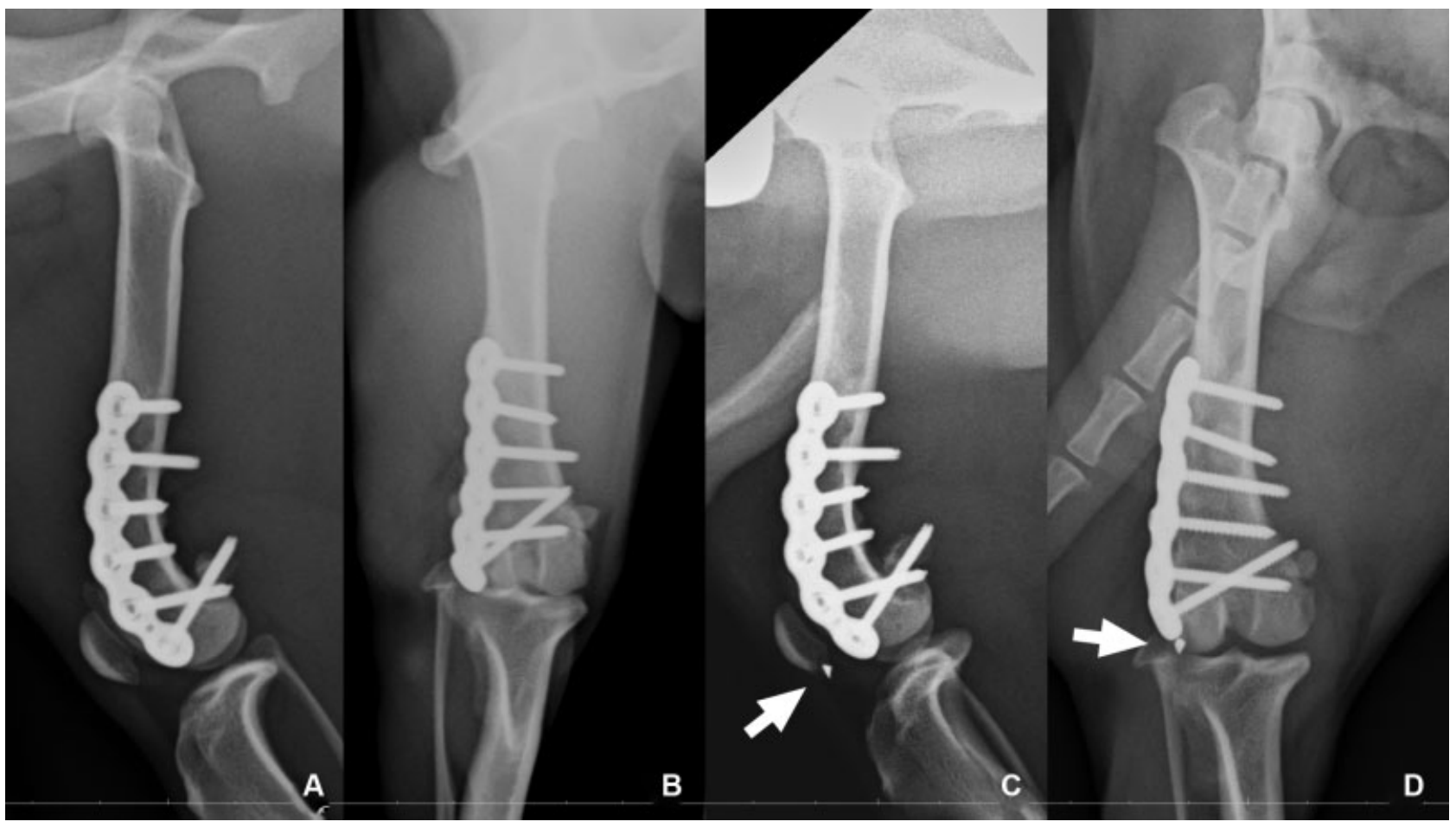

Fig. 2 Jack Russell Terrier, male, 1 year old. Three-month radiographic examination. Mediolateral (A) and craniocaudal (B) radiographs of the right femur showing bone healing. Jack Russell Terrier, male, 16 months old. Seven-month radiographic examination mediolateral (C) and craniocaudal (D) radiographs of the right femur showing the foreign body (arrows). 
Postoperative radiographs showing implant removal were missing.

There was no apparent skin wound or redness over the stifle joint, and no trauma was reported by the owners. Orthopaedic examination revealed pain upon stifle palpation or joint motion and mild swelling of the stifle. The drawer test and the tibial compression test were negative. A provisional diagnosis of nonspecific synovitis was established. General haematological and biochemical tests showed no abnormalities. Plain stifle radiographs were obtained under general anaesthesia. Mediolateral or caudocranial orthogonal radiographs showed a distinct small metal-dense foreign body in the region of the medial tibiofemoral joint ( $\mathbf{- F i g}$. 3A, B). The radiographs revealed that the two most proximal screws were left in situ after implant removal which occurred 5 years earlier. The patient was prepared for arthroscopic foreign body removal. Radiographs just before arthroscopy confirmed that the foreign body had not changed position. The stifle joint was inspected through a lateral parapatellar arthroscopic portal, just lateral to the patellar tendon and distal to the distal pole of the patella utilizing a $2.3 \mathrm{~mm} 30$-degree fore-oblique arthroscope. Synovitis was present. Trochlear groove and patella seemed normal. Lateral femoral and tibial condyles, as well as the cruciate ligaments and the menisci, seemed uninjured. The cranial and caudal cruciate ligaments were probed and assessed for stability. The small foreign body was identified at the medial tibiofemoral joint partially sunk into the tibial cartilage. (-Fig. 4A, C). Chondral lesions were identified at both tibial and femoral medial condyles ( $\mathbf{F i g}$. 4B, D). The foreign body was identified as being a broken Torx-type screwdriver tip. During arthroscopic surgery, the foreign body was changing position and was dislodged because of the flushing liquid's turbulence. The foreign body was extracted using an alligator grasper (-Fig. 4E, F). The complete removal of the fragment was confirmed radiographically (-Fig. 3C) The dog was discharged from the hospital the same day and was weight-bearing. The patient's postoperative course was unremarkable and recovery to full weightbearing and pain-free full range of motion occurred within 3 days. ${ }^{15}$ At 2, 4 and 8 weeks postoperative follow-ups, the dog showed no further signs.

\section{Discussion}

Given a clear indication for surgical foreign body removal, this could have performed either arthroscopically or through open arthrotomy. Arthroscopy was preferred. Advantages of arthroscopy included decreased morbidity through miniinvasiveness, rapid recovery, decreased complication rates, improved functional outcomes, shorter anaesthesia and surgery times, possibility for day-hospital care and enhanced client satisfaction. ${ }^{16-22}$ Disadvantages include the relatively high level of skill required, the high cost of the equipment and relatively high costs to the client.

Arthroscopy has been recognized as an efficient means for foreign bodies removal from the stifle joint in human surgery. 5,8-12,14,23 Multiple attempts to arthroscopically remove a broken tip of a curette from an elbow joint failed in a dog. ${ }^{7}$ The fragment then migrated into the radial fossa of the humeral condyle and was left in situ. The dog made an uneventful recovery and, 6 months after surgery showed

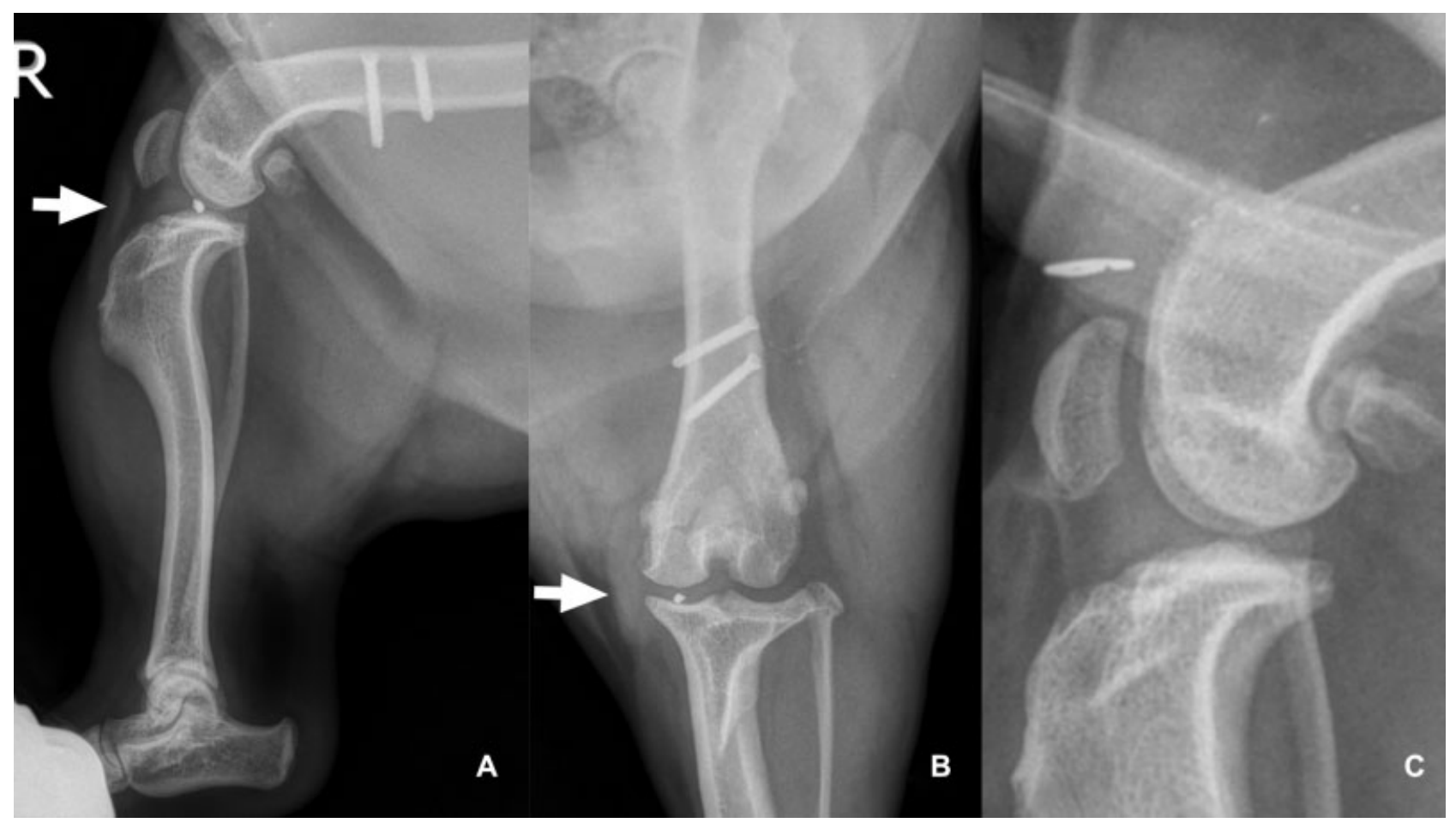

Fig. 3 Jack Russell Terrier, male, 7 years old. Preoperative foreign body arthroscopic removal mediolateral (A) and craniocaudal (B) views of the right femur showing the tibiofemoral joint space location (arrows). Mediolateral view of the right stifle after fragment removal (C). 


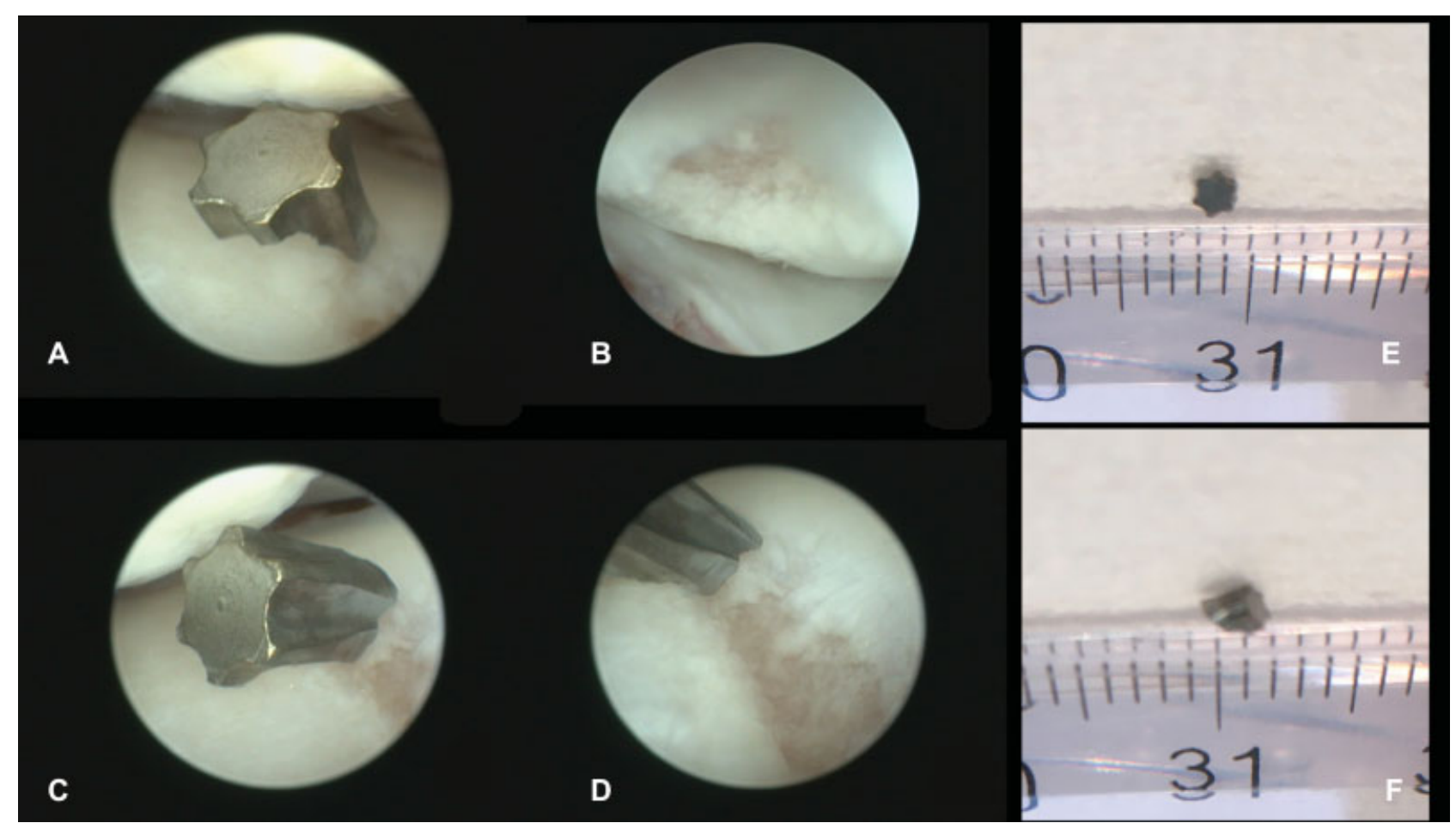

Fig. 4 Arthroscopic images of the foreign body $(A, C)$ and femoral and tibial chondral lesions, respectively (B, D). Images of the screwdriver fragment measurement (E, F).

no lameness except after rest, and there was no evidence of later migration of the metallic fragment. ${ }^{7}$ In our case, the foreign body remained clinically silent for more than 6 years before producing clinical signs. In case of a foreign body left in situ, clinical examination and radiographic inspection should be performed routinely for early detection of potential damages. Foreign bodies can change position over time and sometimes cause delayed complications, as in our case. $^{3,10}$

Plain radiographs were able to detect the foreign body 7 months after surgery. The patient in this case had remained with no evident signs for about 6 years.

Grasping the fragment was technically difficult. The foreign body was continuously changing position because of the ingress-fluid turbulence within the relatively large joint space and because of the force of gravity on the small metallic object. The dislodged piece may migrate to the caudal joint compartment facilitated by the supine position of the dog for arthroscopy (dorsal recumbency). Controlling or closing fluid flow can help during searching for and grasping the foreign body.

In the knee of a human patient, a magnet was used to catch the fragment of a broken sewing needle and to deliver it into the anterior compartment during the removal attempt. ${ }^{10}$ However, we failed to know whether the metal of the screwdriver piece would have acted like a ferromagnetic material.

To ensure that the position of the metal fragment had not changed, radiographs were repeated. Intraoperative X-ray or fluoroscopic examination could be of help in case of migrating foreign bodies. ${ }^{1,3,24}$
The fragment location did not look questionable on our radiographs. In case of doubt, a computed tomography should be performed to detect the exact location just before surgery.

The screwdriver fragment was neither visible on the postoperative radiographs nor at the 3-month radiographic examination (-Fig. 2A, B). The fragment was first detected at the 7month radiographic examination when implant removal was performed ( - Fig. 2C, D). We presume that the screwdriver tip broke during fracture plating and remained stuck within the screw-head recess until later migration through which the fragment became evident at the 7-month postoperative radiographs (-Fig. 2C, D).

The synovitis was probably secondary to cartilage wear at the tibial and femoral medial condyles, or it was secondary to the mechanic irritation from the metallic foreign body.

Intra-articular foreign bodies usually provoke remarkable inflammation. Often neutrophils are the first subpopulation of leukocytes that appear at the injury site, and neutrophil invasion is followed by a sequential increase in the concentrations of macrophage subpopulations..$^{25,26}$ Polymorphonuclear neutrophils are the first cells to take part in the local reactions to the foreign body at the aseptic knee. The local reactions caused by foreign bodies vary widely. ${ }^{10,26}$ In the case of intra-articular sterile foreign bodies, as in our case report, laboratory tests might be unhelpful. On admission, general haematological and biochemical tests showed no abnormalities. Maybe because there was no infection, and the acute phase of the inflammation was short and limited to the joint compartment, laboratory tests remained in the normal range. 
Why and how the screwdriver tip broke, why the two most proximal screws were left in situ after implant removal and why the metal fragment was not removed during the procedure for implant removal is not known. The operative report was missing.

A high index of suspicion for such iatrogenic complications should be maintained even a long time after surgery.

\section{Funding}

None.

\section{Conflict of Interest}

None declared.

\section{Acknowledgments}

The author would like to acknowledge Gerard Mangiagalli, Clinica Veterinaria Europea, Milano, and Francesca Briotti, Ambulatorio Veterinario Venini, Milano, for their contribution to the manuscript and their helpful comments on it.

\section{References}

1 Yeung Y, Wong JK, Yip DK, Kong JK. A broken sewing needle in the knee of a 4-year-old child: is it really inside the knee? Arthroscopy 2003;19(08):E18-E20

2 Brisson BA, Bersenas A, Etue SM. Ultrasonographic diagnosis of septic arthritis secondary to porcupine quill migration in a dog. J Am Vet Med Assoc 2004;224(09):1467-1470, 1453-1454

3 Oztekin HH, Aslan C, Ulusal AE, Sertoz SZ, Ustaoglu RG, Fowler J. Arthroscopic retrieval of sewing needle fragments from the knees of 3 children. Am J Emerg Med 2006;24(04):506-508

4 Barry SL, Lafuente MP, Martinez SA. Arthropathy caused by a lead bullet in a dog. J Am Vet Med Assoc 2008;232(06):886-888

5 Boyle S, Talbot JC, Bismil Q, Schilders E. Arthroscopic removal of a plastic soft drink bottle cap in the knee: a case report. Cases J 2010;3:72

6 Bansal M, Heckl F, English K. Retained broken outflow cannula recovered 6 years post-knee arthroscopy. Orthopedics 2011;34 (12): e945-e947

7 Grand JG, Roig JA, de Swarte M. Instrument breakage as a complication of elbow arthroscopy in a dog. Aust Vet J 2014;92 (04):128-131

8 Helito CP, Faria CEN, Bonadio MB, Pecora JR, Camanho GL, Demange MK. An unusual metallic foreign body inside the knee medial femoral condyle. Case Rep Orthop 2014;2014:849020

9 Gupta R, Malhotra A, Sood M, Masih GD. Retained drain after anterior cruciate ligament surgery: a silent threat to an athlete's career: a case report. J Orthop Case Rep 2017;7(04):10-12

10 Tutkus V, Tutkus J, Jablonskiene V, Abaravicius JA, Silove S, Tutkuviene J. Tibial part of anterior cruciate ligament: a host for silent foreign body in the knee. Chin Med J (Engl) 2018;131(15): 1884-1886

11 Lacy K, Cooke C, Cooke P, Tonnos F. Arthroscopic removal of shotgun pellet from within the medial meniscus. Arthrosc Tech 2016;5(01):e27-e32

12 S M S, K AH, R K, Gnanadoss JJ. An unusual case of partial anterior cruciate ligament (ACL) tear secondary to a glass foreign body in an adolescent knee joint. J Orthop Case Rep 2016;6(03):13-15

13 Ji J-H, Shafi M, Kim D-J. Migration of an extra-articular broken stopcock into the knee joint: an unusual complication of knee arthroscopy. Orthop Surg 2014;6(03):249-251

14 Koaban S, Alatassi R, Alogayyel N. A forgotten retained drain inside a knee for 10 years: a case report. Int J Surg Case Rep 2018; 48:83-86

15 Jaegger G, Marcellin-Little DJ, Levine D. Reliability of goniometry in Labrador Retrievers. Am J Vet Res 2002;63(07):979-986

16 Hoelzler MG, Millis DL, Francis DA, Weigel JP. Results of arthroscopic versus open arthrotomy for surgical management of cranial cruciate ligament deficiency in dogs. Vet Surg 2004;33 (02):146-153

17 Thieman KM, Tomlinson JL, Fox DB, Cook C, Cook JL. Effect of meniscal release on rate of subsequent meniscal tears and ownerassessed outcome in dogs with cruciate disease treated with tibial plateau leveling osteotomy. Vet Surg 2006;35(08):705-710

18 Evans RB, Gordon-Evans WJ, Conzemius MG. Comparison of three methods for the management of fragmented medial coronoid process in the dog. A systematic review and meta-analysis. Vet Comp Orthop Traumatol 2008;21(02):106-109

19 Pozzi A, Hildreth BE III, Rajala-Schultz PJ. Comparison of arthroscopy and arthrotomy for diagnosis of medial meniscal pathology: an ex vivo study. Vet Surg 2008;37(08):749-755

20 Plesman R, Gilbert P, Campbell J. Detection of meniscal tears by arthroscopy and arthrotomy in dogs with cranial cruciate ligament rupture: a retrospective, cohort study. Vet Comp Orthop Traumatol 2013;26(01):42-46

21 Ritzo ME, Ritzo BA, Siddens AD, Summerlott S, Cook JL. Incidence and type of meniscal injury and associated long-term clinical outcomes in dogs treated surgically for cranial cruciate ligament disease. Vet Surg 2014;43(08):952-958

22 Franklin SPSK. Arthroscopy. In: Johnston AATK, ed. Veterinary Surgery Small Animal. Vol 1. second ed. St. Louis, MO: Elsevier; 2018:1323-1347

23 Palmers M, Dierickx C, Peene P, Bijnens E. An unusual metallic foreign body in the lateral tibiofemoral compartment. Arthroscopy $2002 ; 18(03): 325-328$

24 Schmiddem U, Hawi N, Suero EM, Meller R. Combined fluoroscopic and arthroscopic detection and removal of a foreign body lost during elective shoulder arthroscopy: a case report. J Orthop Case Rep 2017;7(02):78-81

25 Anderson JM, Rodriguez A, Chang DT. Foreign body reaction to biomaterials. Semin Immunol 2008;20(02):86-100

26 Cohen HC, Joyce EJ, Kao WJ. Biomaterials selectively modulate interactions between human blood-derived polymorphonuclear leukocytes and monocytes. Am J Pathol 2013;182(06):2180-2190 